\title{
Apropiación académica del papel de la innovación en la competitividad Estrategias de aprendizaje para el emponderamiento de los estudiantes Academic appropriation of the role of innovation in competitiveness Learning strategies for student empowerment
}

\author{
Zulmara Virgínia de Carvalho, Augusto Cesar Bezerra Nobre \\ zvcarvalho@gmail.com,zeaugustojr@hotmail.com \\ Escola de Ciências e Tecnologia \\ Universidade Federal do Rio Grande do Norte \\ Natal, Brasil
}

\begin{abstract}
Resumen- El desbordamiento de la producción académica en la dinámica socioeconómica se configura como uno de los principales desafíos de la gestión de la triple hélice $(3 \mathrm{H})$ brasileña. Según Figueiredo y Pinheiro (2017), el hiato entre la producción científica y tecnológica se refleja en la fragilidad de la competitividad del país. Es dentro de ese escenario que está el relato de prácticas del ecosistema tecnológico educativo de la Escuela de Ciencias y Tecnología de la Universidad Federal de Rio Grande do Norte. Con el objetivo de generar una cultura de innovación sostenible que fortalezca la $3 \mathrm{H}$, a partir de la apropiación académica del papel de la innovación en la competitividad, las prácticas de aprendizaje se centran en el protagonismo discente. El análisis de las prácticas evidencia el empoderamiento de los estudiantes: en la formación en Ciencias y Tecnología, el 72\% de los alumnos se apropia del papel de analistas de Ciencia, Tecnología e Innovación, mientras que el 8\% consigue formular estrategias de derecho de autor. En la formación en Ingeniería de Negocios, el pensar en soluciones inéditas para situaciones-problema presentadas sobre el 33\% y alcanza a todo el alumnado en el posgrado.
\end{abstract}

\section{Palabras clave: Aprendizaje; Innovación; Competitividad}

\begin{abstract}
The spillover of academic production in the socioeconomic dynamics is one of the main challenges of the management of the Brazilian triple helix. According to Figueiredo and Pinheiro (2017), the gap between scientific and technological production is reflected in the fragility of the country's competitiveness. It is within this scenario that is the report of practices of the educational technological ecosystem of the School of Science and Technology of the Federal University of Rio Grande do Norte. With the objective of generating a sustainable innovation culture that strengthens the $3 \mathrm{H}$, from the academic appropriation of the role of innovation in competitiveness, the learning practices are centered on student protagonism. The analysis of the practices evidences the empowerment of the students: in Science and Technology training, $72 \%$ of students take over the role of science, technology and innovation analysts, while $8 \%$ can formulate author strategies. In the training in Business Engineering, the thinking in authorial solutions to problem situations presented to over $33 \%$ and reaches all student body in the graduate.
\end{abstract}

Keywords: Learning; Innovation; Competitiveness

\section{INTRODUCCIÓN}

Aunque es una expresión que ha ganado visibilidad y difusión, en la economía brasileña de la III Revolución Industrial, la apropiación social de la innovación todavía sigue siendo frágil tanto por los actores que componen la triple hélice - Universidades, Industrias y Gobiernos (Etzkowitz, 2009) - cuanto y sobre todo por el ciudadano (Rodrigues, Andrade y Carvalho, 2013). En Brasil, la práctica que está en vigor es universidades produciendo para sí, mientras que las empresas licencian tecnologías, aunque haya incentivos gubernamentales de innovación, de manera sistémica, desde 2004.

De 2004 a 2014, los esfuerzos gubernamentales brasileños se reflejaron en el aumento significativo de Instituciones Científicas y Tecnológicas (ICT) y de Núcleos de Innovación Tecnológica (NIT), así como Parques Tecnológicos, de Incubadoras y del número de empresas con grados, asociadas e incubadas (Carvalho et al., 2014). Sin embargo, las acciones no fueron suficientes, este horizonte de 10 años para que Brasil saliera de la condición de economía de renta media, en la cual se encuentra hace 50 años.

Países de renta mediana enfrontan dificultades para competir con economías de bajo coste de producción y altamente exportadores. Entre las razones, estos países presentan altos costos de producción y tienen insuficiente capacidad tecnológica para oponer con los competidores de las economías de alto valor añadido (Figueiredo y Pinheiro, 2017).

El mapeo de la competitividad industrial brasileña entre 2006 y 2017 puede ser analizado en la tabla 1 'trayectoria de los pilares brasileños de competitividad' que se configura como la recopilación de datos sobre los pilares correlatos extraídos de los informes del 'The Global Competitiveness Report. World Economic Forum' (20062017).

La tabla 1 muestra la baja competitividad de Brasil. El pilar 'Capacidad Tecnológica', que se mantuvo estable entre 2006 y 2017, tiene entre sus dimensiones, el análisis de los acuerdos de transferencia de tecnología. Por otro lado, el 
pilar 'Innovación' es uno de los que más sufrió variacióndesde la posición número 37 en el informe 2006-2007, para la posición número 100 en el informe 2016-2017. Entre las dimensiones que son analizadas en este pilar son: la calidad de la investigación científica; la interacción universidad-industria, en actividades de Investigación y Desarrollo (I\&D); disponibilidad de científicos e ingenieros y número de patentes registradas en el Sistema Internacional de Patentes por habitantes. En el pilar 'Instituciones', entre dos docenas de dimensiones, se evalúa la institucionalidad de protección de la propiedad intelectual. En el informe 2014-2015, Brasil alcanzó la posición más alta $\left(41^{\mathrm{a}}\right)$ en la clasificación en el pilar 'Enseñanza Superior', perdiendo más de 40 posiciones de dos años más tarde.

Tabla 1: Trayectoria de los pilares brasileños de competitividad, entre 2006 y 2017. Preparación propia. Fuente: Klaus Schwab, The Global Competitiveness Report. World Economic Forum (20062017).

Pilar / Informe

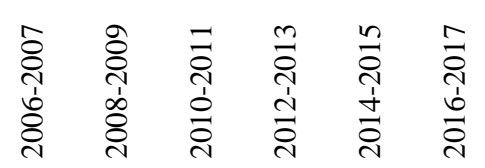

\begin{tabular}{|c|c|c|c|c|c|c|c|}
\hline $1^{\circ}$ & Instituciones & 82 & 91 & 93 & 79 & 94 & 120 \\
\hline $2^{o}$ & Infraestructura & 68 & 78 & 62 & 70 & 76 & 72 \\
\hline $3^{\circ}$ & $\begin{array}{c}\text { Ambiente } \\
\text { Macroeconómico }\end{array}$ & 114 & 122 & 111 & 62 & 85 & 126 \\
\hline $4^{\circ}$ & $\begin{array}{c}\text { Salud y Educación } \\
\text { Básica }\end{array}$ & 59 & 79 & 87 & 88 & 77 & 99 \\
\hline $5^{\circ}$ & $\begin{array}{c}\text { Capacitación y } \\
\text { Enseñanza Superior }\end{array}$ & 56 & 58 & 58 & 66 & 41 & 84 \\
\hline $6^{\circ}$ & $\begin{array}{l}\text { Eficiencia de mercado } \\
\text { de productos }\end{array}$ & 80 & 101 & 114 & 104 & 123 & 128 \\
\hline $7^{\circ}$ & $\begin{array}{c}\text { Eficiencia de } \\
\text { mercado de trabajo }\end{array}$ & 95 & 91 & 96 & 69 & 109 & 117 \\
\hline $8^{\circ}$ & $\begin{array}{c}\text { Desarrollo del } \\
\text { Mercado Financiero }\end{array}$ & 69 & 64 & 50 & 46 & 53 & 93 \\
\hline $9^{\circ}$ & $\begin{array}{l}\text { Capacidad } \\
\text { Tecnológica }\end{array}$ & 54 & 56 & 54 & 48 & 58 & 59 \\
\hline $10^{\circ}$ & Tamaño del mercado & 10 & 10 & 10 & 9 & 9 & 8 \\
\hline $11^{\circ}$ & $\begin{array}{l}\text { Sofisticación de los } \\
\text { Negocios }\end{array}$ & 35 & 35 & 31 & 33 & 47 & 63 \\
\hline $12^{\circ}$ & Innovación & 37 & 43 & 42 & 49 & 62 & 100 \\
\hline
\end{tabular}

Históricamente, los países que lograron escapar de la zona de ingreso de renta media fueron los que aumentaron su productividad, basada en gran medida en la acumulación de capacidad tecnológica para innovación (Figueiredo y Pinheiro, 2017). Como se ve en la tabla 1 , ese es un pilar con poca variación en la serie histórica brasileña 2006-2017, mostrando la mejor posición en el ranking en el informe de 2012-2013 $\left(48^{a}\right)$. Los investigadores todavía defienden que la capacidad tecnológica está almacenada en el capital humano, físico y organizativo que pueden impactar en la productividad y en la inserción internacional de las empresas.

Delante del panorama de baja competitividad debido a la debilidad en el proceso de desbordamiento de la producción científica en la dinámica socioeconómica, en ese artículo cuenta la experiencia de la Escuela de Ciencia y Tecnología (ECT) de la Universidad Federal de Rio Grande del Norte (UFRN) en desarrollar e implementar estrategias de aprendizaje que empoderan al estudiante y lo hace apropiarse del papel de la innovación en la competitividad. Las prácticas objetivan generar una cultura de innovación académica, teniendo en cuenta el artículo 26 de la Ley brasileña $N^{\circ} 10.973$ de 2004, la Ley de la Innovación: "Ias Instituciones Científicas y Tecnológicas que contemplan la enseñanza entre sus actividades principales, deberán asociar; obligatoriamente, la aplicación del dispuesto en esta Ley a las acciones de formación de recursos humanos bajo su responsabilidad".

\section{CONTEXTO}

En Brasil, para la percepción pública de los estudiantes de la educación básica, las humanidades están lejanas de la ingeniería. Concepto que tradicionalmente se mantiene durante la formación tecnológica (Scrignoli et al., 2016). Por ser un agente de aplicación de las ciencias exactas, el ingeniero se define comúnmente como un profesional de carácter puramente técnico. En este contexto, tanto los graduados de los cursos exactos como los cursos de ingeniería tienen una escasa comprensión de su papel en la sociedad, tales como vector de desarrollo socioeconómico.

Sin embargo, en la transición de la III a la IV Revolución Industrial (Schwab, 2016), en el contexto de economías debilitadas, una de las principales estrategias de desarrollo socioeconómico atraviesa por la formación no solo con conocimientos técnicos, sino, sobre todo, los profesionales conscientes del papel de la innovación en la dinámica económica y de las herramientas para llevar a cabo la iniciativa empresarial, como también sean capaces de utilizar la formación aprendida para resolver los problemas contemporáneos. Alineada con esta hipótesis, la famosa inquietud Balzan (1999):

¿Por qué limitarse a transmitir conocimientos si los estudiantes llegan a esto, además de la prensa, inventado más de 500 años, otros medios de acceso a la información? ¿Por qué no centrar los debates en torno a cuestiones planteadas con los estudiantes? ¿Por qué no honrar la compra de mentes creativas y curiosas a través de discusiones, se extrae problemas resoluciones de su propia realidad socio-cultural? ,

prueba, además de la viabilidad, la importancia del protagonismo del estudiante en el proceso de Enseñanza y Aprendizaje (E-A).

Es en este contexto que la Escuela de Ciencia y Tecnología (ECT) - unidad académica responsable por aproximadamente el $10 \%$ de los casi 45.000 estudiantes de la Universidad Federal de Rio Grande do Norte (UFRN) desarrolla y aplica estrategias de enseñanza-aprendizaje en sus cursos de grado y posgrado. Las prácticas ejercitan el análisis en el campo de la Ciencia, Tecnología, Innovación y Sociedad.

Así, objetivando deshacer la percepción de adherencia débil entre el conocimiento de la ciencia humana y el desarrollo tecnológico, se analiza aquí el papel de la cultura de la innovación y el espíritu emprendedor en la sociedad, manteniendo el diálogo entre diversas áreas del conocimiento. 
A partir de la discusión introductoria sobre la interdependencia entre ciencia, tecnología, e innovación y sus reflejos sociales, conceptos de economía, administrativo, social, ambiental y las políticas son abordados y correlacionados a las aplicaciones científicas-tecnológicas y a la generación de negocio de tecnología.

En este proceso, además del discente desarrollar el sentimiento de pertenencia a los procesos de desarrollo socioeconómicos, incluyendo los relacionados a la competitividad internacional, ese estudiante entiende que el cambio de la dinámica económica pasa por la aplicación estratégica del conocimiento académico asociado al análisis de escenarios sociales. Para lograrlo, las prácticas son centradas en el plan conceptual, en análisis de situaciones problemáticas y en la formulación de estrategias para solucionarlas. Aún dentro de las metodologías utilizadas, están en uso de herramientas de enseñanza a distancia, talleres basados en películas y orientaciones a la investigación desde el primer año de carreras de grado.

Dentro del propósito poner a la vista que la acción integrada enseñanza-investigación puede establecer el aula en un laboratorio vivo, incluso en contexto de grandes grupos, así como examinar si la capacidad de construir soluciones rentables y/o disruptivo se lleva a cabo de manera efectiva con la estrategia aplicada por los objetivos planteados en la formación permanente de los estudiantes, se analizaron dos instrumentos de evaluación activos: análisis crítico y el informe de investigación, de los estudiantes: (1) el primer año de la base común de los cursos de ciencia y tecnología; (2) los egresados del último año de curso de énfasis de ingeniería de negocios y (3) los posgrados en ciencia, tecnología e innovación de la Facultad de Ciencia y Tecnología.

Para llevar a cabo este estudio de caso, se analizó de manera cualitativa los instrumentos de evaluación ya dichos, con el fin de identificar la habilidad del estudiante para identificar la innovación como determinante de superación de los estrechamientos socioeconómicos, así como presentar ventanas de oportunidades y proponer soluciones viables, sea desde la búsqueda de soluciones disponibles en la sociedad o en el mercado, sea desde el desarrollo de una idea disruptivo.

\section{DESCRIPCIÓN}

Creada en 2008, ECT tuvo como primera misión el apoyo académico a las actividades del curso de Ciencia y Tecnología - Licenciatura (LCT). El curso fue el tercero en el país a obtener viabilidad a la formación de más de un ciclo, como hecho en el Proceso de Bologna, como muestra en la Tabla 2. LCT ejecuta sus funciones en régimen de grupos grandes (más o menos 140 estudiantes), sumando más de 100 profesores, entre titulares y sustitutos interinos, además de 26 personal administrativo.

Durante la formación interdisciplinaria de núcleo común (Tabla 2), las estrategias de difusión de la cultura de innovación son aplicadas en la asignatura Gestión y Economía de Ciencia, Tecnología e Innovación (GECTI). Así, alrededor del $10 \%$ de los egresos de UFRN son presentados al papel de la interdependencia entre Ciencia, Tecnología e Innovación en la dinámica económica. En concreto, todos los egresados de Ingeniería Ambiental, Biomédica, Computadores, Materiales,
Petróleo, Telecomunicaciones, Mecánica y de Mecatrónica son sensibilizados sobre sus papeles como vectores de cambio socioeconómico. Entre las opciones de formación interdisciplinaria dirigidas a LCT, está el énfasis en Ingeniería de Negocios, que objetiva generar capital intelectual capaz de suscitar riqueza socioeconómica por medio del conocimiento académico. Entre las asignaturas que integran, está la de Fundamentos de Propiedad Intelectual y Transferencia de Tecnología (FPITT), que cede a UFRN el estatus de una de las pocas instituciones brasileñas que aborda tales conocimientos en los cursos de grado.

Tabla 2: Formación en Ciencias y Tecnología - Licenciatura (LCT) en dos ciclos.

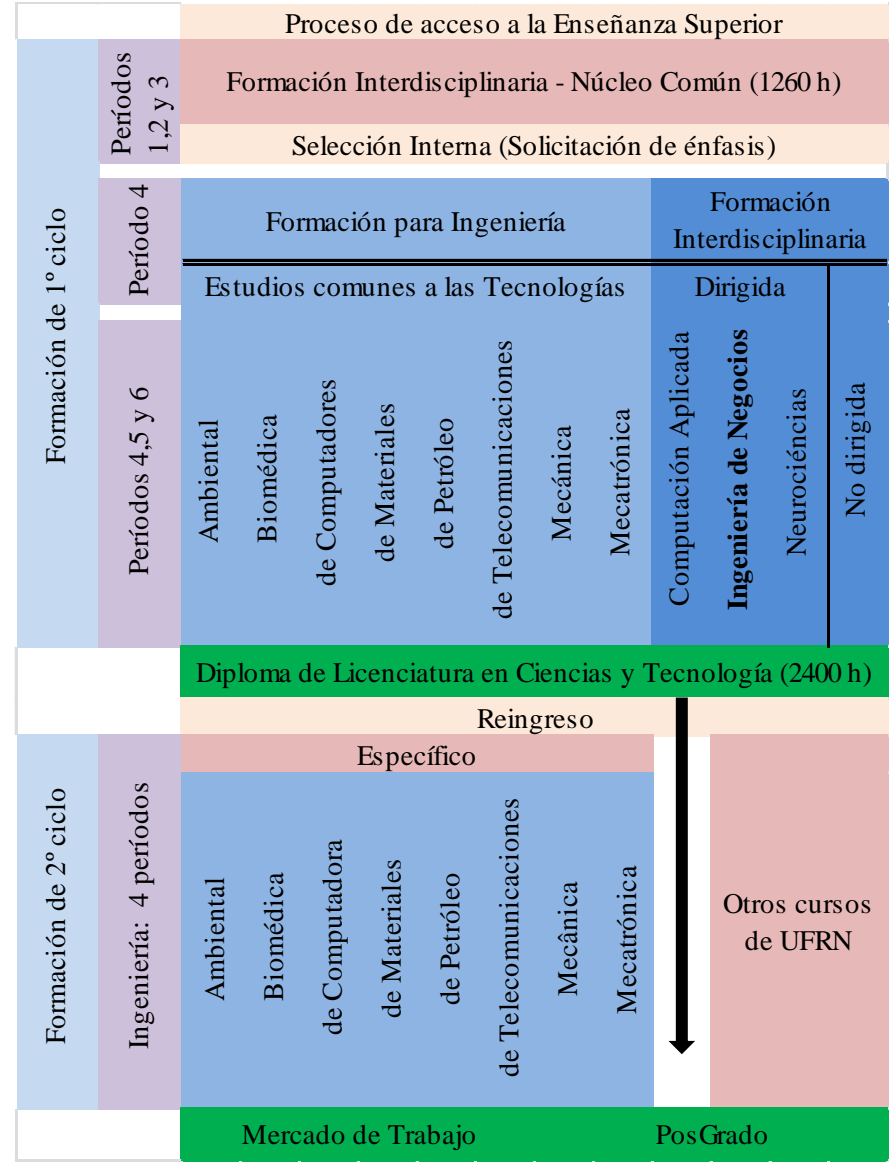

En el campo del posgrado, ECT tiene el Programa de Posgrado en Ciencias, Tecnología y Innovación (PPgCTI), desde 2014, con dos líneas de investigación: gestión de la innovación y desarrollo de tecnologías para la innovación.

Sea en el comienzo de LCT o en la formación interdisciplinaria Ingeniería de Negocios y con más intensidad en PPgCTI, los estudiantes son fuertemente estimulados a convertir sus ideas en negocios con el apoyo de la Incubadora de Procesos Académicos Científicos y Tecnológicos Aplicados (inPACTA), la incubadora proyectos y startups de ECT.

\section{RESUltados}

A Instrumento Evaluativo Análisis Crítico (AC) 
Para dimensionar el nivel de apropiación discente sobre el papel de la innovación en la economía, fueron analizados dos conjuntos de Análisis Crítico (AC) de GECTI. El instrumento evaluativo pretende ejercitar la capacidad de los estudiantes en relacionar la fundamentación teóricaconceptual, con el desarrollo de estrategias innovadoras para situaciones-problema reales.

El análisis crítico del semestre 2016.2 tenía como cuestionamiento fundamental la trayectoria de las políticas de catching up tecnológico brasileño, en el que, por medio de las noticias que estaban basados en conceptos implicando la conectividad entre los actores institucionales del sistema de la ciencia, tecnología e innovación en el país, se buscaba del estudiante la presentación de posibles estrategias para transformar el conocimiento en riqueza socioeconómica en Brasil.

Ya el análisis crítico del semestre 2017.1, desde el escenario de la industria biomédica y el desarrollo de innovaciones en el área, así como la realización de un evento local de la cultura maker, buscaban la elucidación de la interdependencia entre la ciencia, la tecnología y la innovación, como también la presentación de ventanas de oportunidades e innovaciones-clave en el sector industrial antes mencionado y los efectos de la expansión de la cultura maker en la dinámica económica del norte-riograndense.

El análisis exploró tres factores que se presentan fundamentales para la construcción de la percepción del estudiante como vector de cambio en el escenario discutido: (1) ¿El estudiante supo presentar la importancia de la innovación como factor de la superación de los obstáculos presentados en la situación-problema?; (2) Dado el escenario presentado, ¿el estudiante investigó y presentó innovaciones en el área? y (3) ¿El estudiante presentó una estrategia de innovación para actuar como solución de la situaciónproblema, de su autoría.

En esta etapa de la carrera de grado, el estudio reveló que el $72 \%$ de los estudiantes puede tomar el papel de analista de la ciencia, la tecnología y la innovación. En adición, el 8\% consiguen desarrollar estrategias de derechos de autor.

Evaluación análoga, hecha en la asignatura FPITT, expresa la apropiación de la cultura de la innovación de las estrategias de derechos de autor por el 33\% de los estudiantes de énfasis de Ingeniería de Negocios. Repitiendo el experimento en la asignatura de Innovación, Universidades y Gobiernos (UIG) del posgrado, los estudiantes son plenamente capaces de formular sus propias estrategias para la solución de situación-problema presentadas. En este contexto, la figura 1 ilustra los niveles de apropiación académica del papel de la innovación en la competitividad frente a cada nivel de formación.

\section{B Acción Integrada: enseñanza e investigación}

En 2017.1, la metodología de E-A activa de la ECT trató de lanzar un desafío para los estudiantes de GECTI, de FPITT y de IUG: en conjuntos de cinco integrantes, bajo cosupervisión del asistente de docencia, desarrollar la investigación científica por medio de la fundamentación teórica-conceptual de la clase delante de recorte temático: Ciudades Inteligentes, sus principales sectores, y los principales retos para su implementación en la ciudad de
Natal, ubicada en el noreste de Brasil. Como incentivo, la oportunidad de tener un artículo aceptado y participar en un evento internacional IEEE sobre el tema, que se celebró a nivel local: First IEEE Summer School on Smart Cities August 6-11, 2017 - Natal, Brazil.

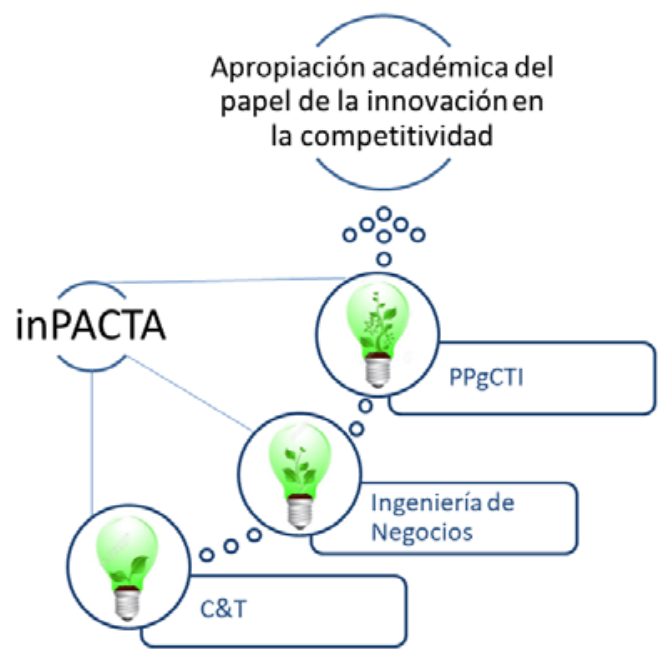

Figura 1: Ecosistema de educación orientado hacia a la Ciencia, Tecnología e Innovación de ECT - UFRN.

Dado que los sectores de las Ciudades Inteligentes dialogan directamente con los aspectos del desarrollo económico, fue ejecutada la investigación, de carácter descriptivo y exploratorio, así como un análisis comparativo entre la trayectoria económica de un país (estudio de caso) y Brasil. En dirección de pensar estrategias de implementación de las Ciudades Inteligentes, la potencialidad de apropiación de tecnologías inteligentes por las ciudades brasileñas fue investigada. En concreto, fueron investigados el desempeño de generación de riqueza; políticas económicas y la balanza de pagos tecnológicos, además de la cultura de innovación de cada país.

Consiguieron desarrollar la investigación y someter el artículo para el evento el 50\% de los grupos de GECTI; el $60 \%$ FPITT y el $100 \%$ IUG.

\section{CONCLUSIONES}

La todavía frágil capacidad en desbordar la producción académica en la sociedad desde las Instituciones CientíficoTecnológicas brasileñas relega al país a un escenario débil estructura económica, con bajas ganancias de competitividad en su cadena productiva.

Este escenario se acentúa en tiempos de crisis económica, en los cuales se evidencian debilidades socioeconómicas locales y disminuye la capacidad de acumulación de riqueza a partir de las bases de productiva en actividad por la falta de liderazgo de los productos locales delante de países de grande competitividad sistémica.

En este contexto, como una relación dialéctica, las bases estructurales de la economía local son puestas en diálogo y urgen soluciones disruptivas que, al mismo tiempo, superan sus limitaciones estructurales, explotan las ventajas comparativas locales con el fin de estimular el crecimiento y el desarrollo económico . 
En frente del reto de aplicación de la innovación como elemento básico para el contexto técnico-económico del siglo XXI, la ECT busca, a través de prácticas innovadoras, permitir a los estudiantes a entender, desarrollar y gestionar la innovación en diversos áreas de cobertura de la triple-hélice, para formar vectores de cambio en diferentes áreas de la formación del discente.

Por lo tanto, y sobre los resultados obtenidos a partir del análisis de los instrumentos de evaluación para los estudiantes del núcleo común, del énfasis ingeniería de negocios y de los estudiantes de posgrados en ciencia, tecnología e innovación, las prácticas de aprendizaje desarrolladas y aplicadas por la ECT empoderan progresivamente el estudiante para llevar a tomar posesión del pensar en los problemas y soluciones en el campo de Ciencia, Tecnología, Innovación y Sociedad, sacándole de la pasividad de la transmisión tradicional del conocimiento.

El análisis del resultado indica que otros trabajos pueden ser desarrollados sobre la base de este estudio de caso, a fin de permitir la escalabilidad y la mejora continua del proceso de enseñanza-aprendizaje desarrollado en ECT, tales como estructuración del método aplicado en las asignaturas de énfasis ingeniería de negocios, como también el desarrollo de la estrategia con el fin de consolidar, ya en el núcleo general, la capacidad de desarrollar soluciones disruptivas.

Teniendo en cuenta lo anterior, la investigación expone que la acción integrada enseñanza-investigación puede establecer el aula en un laboratorio vivo, incluso en contexto de grandes grupos. Entre los resultados, el estudiante es empoderado y se apropia del pensar en soluciones creativas para situaciones-problemáticas, centrándose en el desarrollo socio-económico. En particular, el papel de la innovación en la competitividad. Aunque incipiente, la estrategia de enseñanzaaprendizaje presentada se proyecta como instrumento viable para la difusión de la cultura de la innovación.

\section{REFERENCIAS}

Balzan, N. C. Formação de professores para o ensino superior: desafios e experiências. In: Bicudo MA, organizador. Formação do educador e avaliação educacional. São Paulo: Editora UNESP; 1999. p. 173-88.

Carvalho, Z. V., Bezerra, A. F. A., Silva, W. S. C., Lopes Jr., S. C., Brandão, G. B. Dez anos da lei de inovação: reflexos no cenário brasileiro de parques tecnológicos e incubadoras de empresas. XXIV Seminário Nacional de Parques Tecnológicos e Incubadoras de Empresas. Belém, 2014.

Etzkowitz, H. Hélice tríplice: universidade-indústria-governo: inovação em movimento. EDIPUCRS. Porto Alegre, 2009.

Figueiredo, P. N. E Pinheiro, M. C. Aprendizagem Tecnológica e Inovação Industrial no Brasil. FGV/EBAPE. Rio de Janeiro, 2017.

Presidência Da República. Lei 10.973/2004: dispõe sobre incentivos à inovação e à pesquisa científica e tecnológica no ambiente produtivo e dá outras providências. Presidência da República, Brasília, 02/12/2004.

Rodrigues, R.C., Andrade, B.F. E Carvalho, Z. C. O papel da popularização da inovação no desenvolvimento socioeconômico. Anais do II Encontro Nacional de Popularização da Ciência, Tecnologia e Inovação. Rio de Janeiro, 2013.

Schwab, K. A quarta Revolução Industrial. WEF. Edipro, 2016.

Scrignoli, G.M., Colombo, E. R., Olguin, G. S.. Matai, P. H. L. S. As Ciências Humanas no Curso de Engenharia. Escola Politécnica, Universidade de São Paulo, São Paulo, 2016. 